\title{
Improved Lower Bounds For The Capacitated Lot Sizing Problem With Set Up Times
}

\author{
Zeger Degraeve, Raf Jans
}

\begin{tabular}{|l|l|}
\hline \multicolumn{2}{|l|}{ ERIM REPORT SERIES RESEARCH IN MANAGEMENT } \\
\hline ERIM Report Series reference number & ERS-2003-026-LIS \\
\hline Publication & April 2003 \\
\hline Number of pages & 25 \\
\hline Email address corresponding author & rjans@fbk.eur.nl \\
\hline Address & Erasmus Research Institute of Management (ERIM) \\
& Rotterdam School of Management / Faculteit Bedrijfskunde \\
& Rotterdam School of Economics / Faculteit Economische \\
& Wetenschappen \\
& Erasmus Universiteit Rotterdam \\
& P.O. Box 1738 \\
& 3000 DR Rotterdam, The Netherlands \\
& Phone: $\quad+31104081182$ \\
& Fax: $\quad+3110$ 408 9640 \\
& Email: info@erim.eur.nl \\
& Internet: $\quad$ www.erim.eur.nl \\
\hline
\end{tabular}

Bibliographic data and classifications of all the ERIM reports are also available on the ERIM website: www.erim.eur.nl 


\title{
ERASMUS RESEARCH INSTITUTE OF MANAGEMENT
}

\author{
REPORT SERIES
}

RESEARCH IN MANAGEMENT

\begin{tabular}{|c|c|c|}
\hline \multicolumn{3}{|c|}{ BIBLIOGRAPHIC DATA AND CLASSIFICATIONS } \\
\hline Abstract & \multicolumn{2}{|c|}{$\begin{array}{l}\text { We present new lower bounds for the Capacitated Lot Sizing Problem with Set Up Times. We } \\
\text { improve the lower bound obtained by the textbook Dantzig-Wolfe decomposition where the } \\
\text { capacity constraints are the linking constraints. In our approach, Dantzig-Wolfe decomposition is } \\
\text { applied to the network reformulation of the problem. The demand constraints are the linking } \\
\text { constraints and the problem decomposes into subproblems per period containing the capacity } \\
\text { and set up constraints. We propose a customized branch-and-bound algorithm for solving the } \\
\text { subproblem based on its similarities with the Linear Multiple Choice Knapsack Problem. Further } \\
\text { we present a Lagrange Relaxation algorithm for finding this lower bound. To the best of our } \\
\text { knowledge, this is the first time that computational results are presented for this decomposition } \\
\text { and a comparison of our lower bound to other lower bounds proposed in the literature indicates } \\
\text { its high quality. }\end{array}$} \\
\hline \multirow{3}{*}{$\begin{array}{l}\text { Library of Congress } \\
\text { Classification } \\
\text { (LCC) }\end{array}$} & $5001-6182$ & Business \\
\hline & $5201-5982$ & Business Science \\
\hline & HB 143.7 & Optimization techniques \\
\hline \multirow{4}{*}{$\begin{array}{l}\text { Journal of Economic } \\
\text { Literature } \\
(\mathrm{JEL})\end{array}$} & M & Business Administration and Business Economics \\
\hline & M 11 & Production Management \\
\hline & R 4 & Transportation Systems \\
\hline & C 61 & Optimization techniques; programming models; dynamic analysis \\
\hline \multirow{4}{*}{$\begin{array}{l}\text { European Business Schools } \\
\text { Library Group } \\
\text { (EBSLG) }\end{array}$} & $85 \mathrm{~A}$ & Business General \\
\hline & $260 \mathrm{~K}$ & Logistics \\
\hline & $240 \mathrm{~B}$ & Information Systems Management \\
\hline & $260 \mathrm{~N}$ & Production planning \\
\hline \multicolumn{3}{|c|}{ Gemeenschappelijke Onderwerpsontsluiting (GOO) } \\
\hline \multirow[t]{4}{*}{ Classification GOO } & 85.00 & Bedrijfskunde, Organisatiekunde: algemeen \\
\hline & 85.34 & Logistiek management \\
\hline & 85.20 & Bestuurlijke informatie, informatieverzorging \\
\hline & 85.03 & Methoden en technieken, operations research \\
\hline \multirow[t]{3}{*}{ Keywords GOO } & \multicolumn{2}{|c|}{ Bedrijfskunde / Bedrijfseconomie } \\
\hline & \multicolumn{2}{|c|}{ Bedrijfsprocessen, logistiek, management informatiesystemen } \\
\hline & \multicolumn{2}{|c|}{ Series, capaciteit, productieplanning, modellen } \\
\hline Free keywords & \multicolumn{2}{|c|}{ Capacitated Lot Sizing; Dantzig-Wolfe decomposition; Lagrange Relaxation; Lower Bounds } \\
\hline
\end{tabular}




\title{
IMPROVED LOWER BOUNDS FOR THE CAPACITATED Lot Sizing Problem With Set Up Times
}

\author{
Zeger Degraeve \\ London Business School \\ Regent's Park, London NW1 4SA, U.K. \\ zdegraeve@london.edu \\ Raf Jans* \\ Rotterdam School of Management, Erasmus University \\ PO Box 1738, 3000 DR Rotterdam, The Netherlands \\ rjans@fbk.eur.nl
}

\begin{abstract}
We present new lower bounds for the Capacitated Lot Sizing Problem with Set Up Times. We improve the lower bound obtained by the textbook Dantzig-Wolfe decomposition where the capacity constraints are the linking constraints. In our approach, Dantzig-Wolfe decomposition is applied to the network reformulation of the problem. The demand constraints are the linking constraints and the problem decomposes into subproblems per period containing the capacity and set up constraints. We propose a customized branch-and-bound algorithm for solving the subpıoblem based on its similarities with the Linear Multiple Choice Knapsack Problem. Further we present a Lagrange Relaxation algorithm for finding this lower bound. To the best of our knowledge, this is the first time that computational results are presented for this decomposition and a comparison of our lower bound to other lower bounds proposed in the literature indicates its high quality.
\end{abstract}

\footnotetext{
* Corresponding author. The author was partially funded by the Fund for Scientific ResearchFlanders (F.W.O.), Belgium.
} 


\section{Introduction}

We consider the Capacitated Lot Sizing Problem with Set Up Times (CLST). Let $P$ be the set of products $\{1, \ldots, n\}$ with index $i$ and $T$ the set of time periods $\{1, \ldots, m\}$ with index $t$. We have the following parameters: $d_{i t}$ is the demand of product $i$ in period $t$; $s d_{i t k}$ is the sum of the demand of product $i$ from period $t$ until period $k ; h c_{i t}$ is the holding cost for product $i$ in period $t ; s c_{i t}$ is the set up cost for product $i$ in period $t$; $v c_{i t}$ is the variable production cost for product $i$ in period $t ; f c_{i}$ is the cost for initial inventory for product $i$; $s t_{i t}$ is the set up time for product $i$ in period $t$; $v t_{i t}$ is the variable production time for product $i$ in period $t$ and $\operatorname{cap}_{t}$ is the capacity in period $t$. We have the following decision variables: $x_{i t}$ is the amount of production of product $i$ in period $t ; y_{i t}=1$ if there is a set up for product $i$ in period $t,=0$ otherwise and $s i_{i}$ is the amount of initial inventory for item $i$. The mathematical formulation of the CLST is then as follows:

$$
\begin{aligned}
& \operatorname{Min} \sum_{i \in P} f c_{i} s i_{i}+\sum_{i \in P} \sum_{t \in T}\left(s c_{i t} y_{i t}+v c_{i t} x_{i t}+h c_{i t} s_{i t}\right) \\
& \text { s.t. } \quad s i_{i}+x_{i, 1}=d_{i, 1}+s_{i, 1} \quad \forall i \in P \\
& \left.s_{i, t-1}+x_{i t}=d_{i t}+s_{i t} \quad \forall i \in P, \forall t \in T \backslash 1\right\} \\
& x_{i t} \leq \min \left\{\left(c_{a p}-s t_{i t}\right) / v t_{i t}, s d_{i t m}\right\} y_{i t} \quad \forall i \in P, \forall t \in T \\
& \sum_{i \in P}\left(s t_{i t} y_{i t}+v t_{i t} x_{i t}\right) \leq c a p_{t} \quad \forall t \in T \\
& y_{i t} \in\{0,1\}, x_{i t} \geq 0, s_{i t} \geq 0, s i_{i} \geq 0 \quad \forall i \in P, \forall t \in T
\end{aligned}
$$

The objective function (1) minimizes the total costs, consisting of the initial inventory cost, the set up cost, the variable production cost and the inventory holding cost. Constraints (2.1) and (2.2) are the demand constraints. To deal with infeasible problems, we allow for initial inventory which is available in the first period at a large feasibility cost of $f_{c}$. Constraint (3) is the set up forcing constraint: if there is any positive production in period $t$, a set up is enforced. In order to make the formulation stronger, we limit the production for each item by both the remaining demand and the maximum possible production with the available capacity minus the set up time. Next, there is a constraint on the available capacity in each period (4). If we have a set up, 
the set up time is properly accounted for. Finally, there are the non-negativity and integrality constraints (5). Let $v_{C L S T}$ be the optimal objective value for problem (1)-(5) and $\bar{v}_{C L S T}$ for its LP relaxation. This problem can be reformulated using the variable redefinition approach of Eppen and Martin [6]. Define the following parameters:

$c v_{i t k}$ : total production and holding cost for producing item $i$ in period $t$ to satisfy demand for the periods $t$ until $k$,

$$
=v c_{i t} s d_{i t k}+\sum_{s=t+1 u=t}^{k} \sum_{i u}^{s-1} h c_{i s},
$$

$c i_{i t} \quad:$ total production and holding cost for initial inventory for product $i$ to satisfy demand from period 1 up to period $t$,

$$
=f c_{i} s d_{i l t}+\sum_{s=2}^{t} \sum_{u=1}^{s-1} h c_{i u} d_{i s} .
$$

We also have the following new variables in our model:

$z v_{i t k}$ : fraction of the production plan for product $i$ where production in period $t$ satisfies demand from period $t$ to period $k$,

$w_{i t} \quad$ : fraction of the initial inventory plan for product $i$ where demand is satisfied for the first $t$ periods.

The network reformulation is then as follows:

$$
\begin{aligned}
& \operatorname{Min} \sum_{i \in P} \sum_{t \in T}\left(s c_{i t} y_{i t}+c i_{i t} w_{i t}\right)+\sum_{i \in P} \sum_{t \in T} \sum_{k=t}^{m} c v_{i t k} z v_{i t k} \\
& \text { s.t. } 1=\sum_{k \in T}\left(w_{i k}+z v_{i, 1, k}\right) \quad \forall i \in P \\
& \left.w_{i, t-1}+\sum_{k=1}^{t-1} z v_{i k, t-1}=\sum_{k=t}^{m} z v_{i t k} \quad \forall i \in P, \forall t \in T \backslash 1\right\} \\
& \sum_{k=t}^{m} z v_{i t k} \leq y_{i t} \quad \forall i \in P, \forall t \in T \\
& \sum_{i \in P} s t_{i t} y_{i t}+\sum_{i \in P} \sum_{k=t}^{m} v t_{i t} s d_{i t k} z v_{i t k} \leq \operatorname{cap}_{t} \quad \forall t \in T \\
& y_{i t} \in\{0,1\}, w_{i t} \geq 0 \quad \forall i \in P, \forall t \in T \\
& z v_{i t k} \geq 0 \quad \forall i \in P, \forall t \in T, \forall k \in T, k \geq t
\end{aligned}
$$

The objective function (6) minimizes the sum of the set up cost, initial inventory cost and regular production and holding costs. Constraints (7) and (8) define the flow 
constraints for the shortest path network. For each product, a unit flow is sent through the network, imposing that the demand for each product has to be satisfied without backlogging. Figure 1 represents the network for a three period problem for a specific item $i$.

Figure 1. Network representation for one item and 3 periods

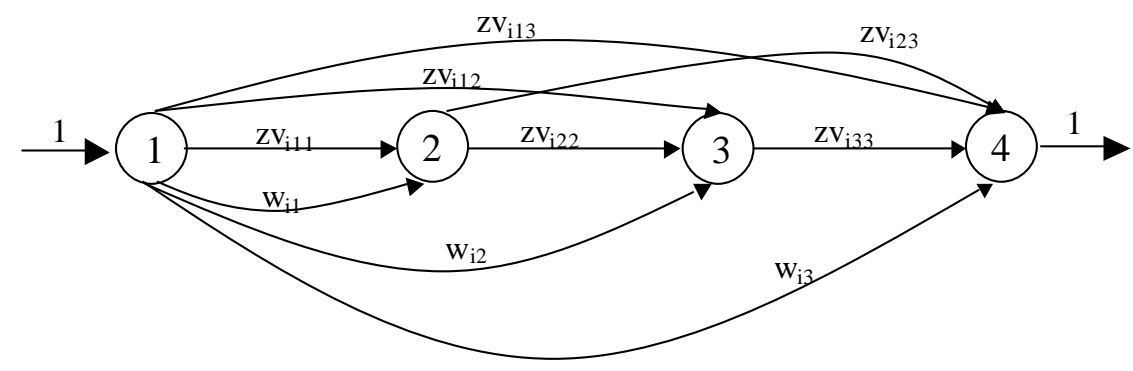

Constraint (9) defines the set up forcing for each item. The capacity constraints (10) limit the sum of the total set up times and production times to the available capacity in each period. The set up decision is binary (11). Let $v_{E M}$ be the optimal objective value of the Eppen and Martin reformulation (6)-(12) and $\bar{v}_{E M}$ the LP relaxation value.

\section{Decomposition Approaches}

\subsection{Overview of Different Bounds}

In the traditional textbook Dantzig-Wolfe decomposition for CLST, the capacity constraints are the linking constraints and the demand and set up constraints and the integrality conditions are put in the subproblem. The problem decomposes into uncapacitated lot sizing subproblems per item. For the regular formulation (1)-(5), this gives us the lower bound $\bar{v}_{C L D 1}[5,9]$. We discuss a new decomposition, where the demand constraints are the linking constraints. The problem decomposes into subproblems per time period containing the capacity and set up constraints and the integrality conditions. The columns are production plans per period, indicating for each period which products are produced and in what quantities. All these production 
plans are feasible with respect to the capacity constraint. To the best of our knowledge, Diaby et al. [4] are the only researchers to test such a decomposition per time period, but they start from the formulation (1)-(5), giving the lower bound $\bar{v}_{C L D 2}$. From their computational tests, they conclude that the decomposition per item is superior to the decomposition per time period. In this paper, we start from the network formulation and applying the decomposition per time period results in the lower bound $\bar{v}_{E M D 2}$. This bound is discussed, without computational results, for the Capacitated Lot Sizing Problem (CLSP) without Set Up Times in Chen and Thizy [3]. An overview of these different lower bounds is given in Table 1. Propositions 1, 2 and 3 state the relationship between these bounds. Note that $\bar{v}_{C L D 1}$ is also equal to the regular formulation (1)-(5) plus the $(l, S)$-inequalities [1].

Table 1. Overview of different lower bounds for CLST

\begin{tabular}{l|ccc} 
& No decomposition & $\begin{array}{c}\text { Decomposition 1 } \\
\text { (per item) }\end{array}$ & $\begin{array}{c}\text { Decomposition 2 } \\
\text { (per time period) }\end{array}$ \\
\hline $\begin{array}{l}\text { Regular formulation } \\
\text { (1)-(5) }\end{array}$ & $\bar{v}_{C L S T}$ & $\bar{v}_{C L D 1}$ & $\bar{v}_{C L D 2}$ \\
$\begin{array}{l}\text { Network formulation } \\
(6)-(12)\end{array}$ & $\bar{v}_{E M}$ & $\bar{v}_{E M D 1}$ & $\bar{v}_{E M D 2}$
\end{tabular}

Proposition 1: $\bar{v}_{C L S T} \leq \bar{v}_{C L D 1}=\bar{v}_{E M}=\bar{v}_{E M D 1} \leq \bar{v}_{E M D 2}$

Proof: The first inequality follows from the fact that applying Dantzig-Wolfe decomposition to a minimization problem can never decrease the lower bound. The subproblem is the single item uncap acitated lot sizing problem [15] and the regular formulation of this problem in the $\left(x_{i t}, s i_{i}, s_{i t}, y_{i t}\right)$-space does not have the integrality property. The equivalence between $\bar{v}_{C L D 1}$ and $\bar{v}_{E M}$ is established by the fact that both formulations describe the convex hull of the single item uncapacitated lot sizing problems. Further, applying the decomposition per item to the network reformulation will not improve the lower bound, as the subproblem is the network reformulation of the single item uncapacitated lot sizing problem and it has the integrality property [6]. This establishes the equivalence between $\bar{v}_{E M}$ and $\bar{v}_{E M D 1}$. Finally, applying the 
decomposition per period to the network formulation will lead to an equal or better lower bound, as the subproblem does not have the integrality property. Q.E.D.

Proposition 2: $\bar{v}_{C L S T} \leq \bar{v}_{C L D 2} \leq \bar{v}_{E M D 2}$

Proof: The first inequality follows from the fact that applying Dantzig-Wolfe decomposition to a minimization problem can never decrease the lower bound and the subproblem does not have the integrality property. The proof for the second inequality is similar to a proof provided by Chen and Thizy [3] for the Capacitated Lot Sizing Problem without Set Up Times. A feasible point $\left(z v_{i t k}, w_{i t}, y_{i t}\right)$ of the network model with the decomposition per period satisfies the network constraints (7) and (8) and is contained in the convex hull of (9)-(12). The corresponding point in the $\left(x_{i t}, s i_{i}, s_{i t}, y_{i t}\right)$-space will satisfy the demand constraints (2.1) and (2.2) and will be contained in the convex hull of (3)-(5). The reverse is not true. Details of this proof can be found in the appendix. Q.E.D.

Proposition 3: The bound $\bar{v}_{C L D 1}$ does not dominate $\bar{v}_{C L D 2}$ or vice versa.

Proof: Chen and Thizy [3] provide an example that the bound $\bar{v}_{C L D 1}$ does not dominate $\bar{v}_{C L D 2}$ or vice versa for the CLSP without set up times, which is a special case of the general problem with set up times. Q.E.D.

\subsection{Dantzig-Wolfe Reformulation and Lagrange Relaxation}

We focus on the decomposition of the network reformulation into subproblems per time period, containing the set up and capacity constraints. For this new decomposition, define $S_{t}$ as the set of all the extreme point production plans of the subproblem for period $t$. The variable $z t_{t q}$ is associated with production plan $q$ for period $t$. The master program is then as follows:

$$
\begin{array}{lll} 
& \operatorname{Min} \sum_{i \in P} \sum_{t \in T} c i_{i t} w_{i t}+\sum_{t \in T} \sum_{q \in S_{t}} c t_{t q} z t_{t q} & \\
\text { s.t. } & 1=\sum_{k \in T}\left(w_{i k}+\sum_{q \in S_{1}} a_{i 1 k q} z t_{1 q}\right) & \forall i \in P
\end{array}
$$




$$
\begin{array}{lll}
w_{i, t-1}+\sum_{k=1}^{t-1} \sum_{q \in S_{k}} a_{i k, t-1, q} z t_{k q}=\sum_{k=t}^{m} \sum_{q \in S_{t}} a_{i k k q} z t_{t q} & & \\
\sum_{q \in S_{t}} z t_{t q}=1 & \forall t \in P, \forall t \in T \backslash 1\} & \pi_{i t} \\
z t_{i q} \geq 0 & & \mu_{t}
\end{array}
$$

The objective function (13) minimizes the total cost of the initial inventory and the cost of the production plans chosen in each period. Constraints (14) and (15) are the flow constraints and correspond to the constraints (7) and (8) in the original formulation. The flow constraints have a dual price $\pi_{i t}$. The convexity constraints (16) have a dual price $\mu_{t}$. The constraint coefficient parameters $a_{i t k q}$ and the cost parameter $c t_{t q}$ of the $z t_{t q}$ variables are defined by the solution of the subproblem. In the subproblems, the objective function minimizes the reduced cost. For each time period $t$, except for the last one, we have the following objective function:

$$
\operatorname{Min} \sum_{i \in P} s c_{i t} y_{i t}+\sum_{i \in P} \sum_{k=t}^{m} c v_{i t k} z v_{i t k}-\sum_{i \in P} \sum_{k=t}^{m} \pi_{i t} z v_{i t k}+\sum_{i \in P} \sum_{k=t}^{m-1} \pi_{i, k+1} z v_{i t k}-\mu_{t}
$$

After rearranging the terms in the objective function, the subproblem for period $t \mathrm{can}$ be stated as follows:

$$
\begin{array}{lll} 
& \operatorname{Min} \sum_{i \in P} s c_{i t} y_{i t}+\sum_{i \in P} \sum_{k=t}^{m-1}\left(c v_{i t k}-\pi_{i t}+\pi_{i, k+1}\right) z v_{i t k}+\sum_{i \in P}\left(c v_{i t m}-\pi_{i t}\right) z v_{i t m}-\mu_{t} \\
\text { s.t. } \quad & \sum_{i \in P} s t_{i t} y_{i t}+\sum_{i \in P} \sum_{k=t}^{m} v t_{i t} s d_{i t k} z v_{i t k} \leq c a p_{t} & \\
& \sum_{k=t}^{m} z v_{i t k} \leq y_{i t} & \forall i \in P \\
y_{i t} \in\{0,1\}, z v_{i t k} \geq 0 & \forall i \in P, \forall k \in T, k \geq t
\end{array}
$$

The constraints in the subproblem are the capacity constraint (20), set up constraints (21) and integrality constraints (22) for period $t$. The objective function for the subproblem of the final period is as follows:

$$
\operatorname{Min} \sum_{i \in P} s c_{i m} y_{i m}+\sum_{i \in P}\left(c v_{i m m}-\pi_{i m}\right) z v_{i m m}-\mu_{m}
$$


During the column generation process, we generate the columns as they are needed. Let $\left(y_{i t}^{*}, z v_{i t k}^{*}\right)$ be the optimal solution for the subproblem for period $t$. The column prices out if the minimum reduced cost (19) is smaller than zero. The new column $q \in S_{t}$ has the following parameters:

$$
\begin{array}{ll}
a_{i t k q}=z v_{i t k}^{*} & \forall i \in P, \forall k \in T, k \geq t \\
c t_{t q}=\sum_{i \in P} s c_{i t} y_{i t}^{*}+\sum_{i \in P} \sum_{k=t}^{m} c v_{i t k} z v_{i t k}^{*} &
\end{array}
$$

The lower bound of this new Dantzig-Wolfe decomposition can also be approximated by Lagrange relaxation. In the Lagrange relaxation reformulation, the network constraints (7) and (8) are dualized in the objective function (6) with non-negative dual multipliers $p_{i t}$ :

$$
\begin{aligned}
\operatorname{Min} & \sum_{i \in P} \sum_{t \in T}\left(s c_{i t} y_{i t}+c i_{i t} w_{i t}\right)+\sum_{i \in P} \sum_{t \in T} \sum_{k=t}^{m} c v_{i t k} z v_{i t k} \\
& -\sum_{i \in P} p_{i 1}\left(\sum_{k \in T} w_{i k}+\sum_{k \in T} z v_{i, 1, k}-1\right) \\
& -\sum_{i \in P} \sum_{t \in T \backslash\{1\}} p_{i t}\left(\sum_{k=t}^{m} z v_{i t k}-w_{i, t-1}-\sum_{k=1}^{t-1} z v_{i k, t-1}\right)
\end{aligned}
$$

After rearranging the terms in the objective function, the Lagrange problem becomes:

$$
\begin{aligned}
& \operatorname{Min} \sum_{i \in P} \sum_{t \in T} s c_{i t} y_{i t}+\sum_{i \in P} \sum_{t \in T \backslash\{m\}}\left(c i_{i t}-p_{i, 1}+p_{i, t+1}\right) w_{i t}+\sum_{i \in P}\left(c i_{i m}-p_{i, 1}\right) w_{i m} \\
& +\sum_{i \in P} \sum_{t \in T \backslash\{m\}} \sum_{k=t}^{m-1}\left(c v_{i t k}-p_{i t}+p_{i, k+1}\right) z v_{i t k}+\sum_{i \in P} \sum_{t \in T}\left(c v_{i t m}-p_{i t}\right) z v_{i t m}+\sum_{i \in P} p_{i 1}
\end{aligned}
$$

s.t. (9), (10), (11), (12).

The Lagrange problem decomposes into separate subproblems for each period $t$ :

$$
\operatorname{Min} \sum_{i \in P} s c_{i t} y_{i t}+\sum_{i \in P} \sum_{k=t}^{m-1}\left(c v_{i t k}-p_{i t}+p_{i, k+1}\right) z v_{i t k}+\sum_{i \in P}\left(c v_{i t m}-p_{i t}\right) z v_{i t m}
$$

s.t. (20), (21), (22)

The last period objective function has a special form:

$$
\operatorname{Min} \sum_{i \in P} s c_{i m} y_{i m}+\sum_{i \in P}\left(c v_{i m m}-p_{i m}\right) z v_{i m m}
$$


The $w_{i t}$ variables are present in the overall Lagrange objective function (27), but not in the objective function of the separate subproblems (28) because they do not appear in the constraints of the subproblem. To minimize the overall Lagrange objective function (27), the value of each $w_{i t}$ variable is determined according to the following decision rule:

$$
\begin{array}{ll}
w_{i t}=1 \text { if }\left(c i_{i t}-p_{i, 1}+p_{i, t+1}\right)<0, w_{i t}=0 \text { otherwise, } & \forall i \in P, \forall t \in T \backslash\{m\} \\
w_{i m}=1 \text { if }\left(c i_{i m}-p_{i, 1}\right)<0, w_{i m}=0 \text { otherwise. } & \forall i \in P
\end{array}
$$

Further, we implicitly consider constraint (7) by setting, for each item, only the initial inventory variable with the most negative objective coefficient equal to one.

In the Lagrange relaxation algorithm [7], a Lagrange problem is solved during several iterations and the Lagrange dual prices $p_{i t}$ are updated by a standard subgradient optimization scheme (32) and (33). Let $p_{i t}^{r}$ be the dual prices at iteration $r$ and let $\left(y_{i t}^{r}, z v_{i t k}^{r}, w_{i t}^{r}\right)$ the optimal solution for the Lagrange problem at step $r$. The optimal objective value (27) for the Lagrange problem at step $r$ is $\bar{v}_{E M L R}\left(p_{i t}^{r}\right)$. In the calculation of $\lambda$ (34), UB is the best known upper bound for the original problem (1)(5) and $\omega$ is initially set to two and is decreased whenever the Lagrange solution $\bar{v}_{E M L R}\left(p_{i t}^{r}\right)$ has failed to improve in a specified number of steps.

$$
\begin{array}{ll}
p_{i 1}^{r+1}=\max \left\{0, p_{i 1}^{r}-\lambda\left(\sum_{k \in T} w_{i k}^{r}+\sum_{k \in T} z v_{i, 1, k}^{r}-1\right)\right\} & \forall i \in P \\
p_{i t}^{r+1}=\max \left\{0, p_{i t}^{r}-\lambda\left(\sum_{k=t}^{m} z v_{i t k}^{r}-w_{i, t-1}^{r}-\sum_{k=1}^{t-1} z v_{i k, t-1}^{r}\right)\right\} & \forall i \in P, \forall t \in T \backslash\{1\} \\
\lambda=\frac{\omega\left(U B-\bar{v}_{E M L R}\left(p_{i t}^{r}\right)\right)}{\sum_{i \in P}\left(\sum_{k \in T} w_{i k}^{r}+\sum_{k \in T} z v_{i, 1, k}^{r}-1\right)^{2}+\sum_{i \in P} \sum_{t \in T \backslash\{1\}}\left(\sum_{k=t}^{m} z v_{i t k}^{r}-w_{i, t-1}^{r}-\sum_{k=1}^{t-1} z v_{i k, t-1}^{r}\right)^{2}}
\end{array}
$$

For any set of positive multipliers $p_{i t}$ it holds that the value $\bar{v}_{E M L R}\left(p_{i t}\right)$ provides a lower bound on the optimal value $v_{E M}$. The best possible Lagrange lower bound is called the Lagrange Dual: $\bar{v}_{E M L D}=\max _{p_{i t} \geq 0}\left(\bar{v}_{E M L R}\left(p_{i t}\right)\right)$. The value of this Lagrange Dual $\bar{v}_{E M L D}$ is equal to the Dantzig-Wolfe lower bound $\bar{v}_{E M D 2}$. We observe the similarities between Dantzig-Wolfe decomposition and Lagrange relaxation. The Lagrange 
subproblem has exactly the same structure as the column generation subproblem. The only difference is the way the dual multipliers are obtained. The $\pi_{i t}$ multipliers are the dual prices given by the current master of the Dantzig-Wolfe reformulation and the $p_{i t}$ multipliers in the Lagrange relaxation are updated with subgradient optimization. As there is no guarantee for convergence and no clear stopping criterion for the Lagrange algorithm, the bound that we obtain with Lagrange Relaxation is only an approximation of the Dantzig-Wolfe lower bound.

\section{Solving the Subproblem}

Next we develop a customized branch-and-bound algorithm for solving the subproblem (19)-(22). The relaxation of the subproblem can be solved with an algorithm for the Linear Multiple Choice Knapsack Problem. The Multiple Choice Knapsack Problem is an extension of the Binary Knapsack Problem where the items are divided into several disjoint classes. The problem is to choose exactly one item from each class so that the total profit is maximized without exceeding the capacity of the knapsack. The relaxation of this problem is called the Linear Multiple Choice Knapsack Problem (LMCKP). Sinha and Zoltners [13] and Pisinger [11] present some basic definitions and properties of the problem. The relaxation of our subproblem (19)-(22) is a LMCKP. The set up constraint (21) becomes an equality constraint in the relaxation as the set up variables have a positive cost $s c_{i t}$ and positive set up time $s t_{i t}$ in this minimization problem. Therefore we can substitute out the set up variables. The subproblem for period $t$ now contains the capacity constraint (36) and the simple upper bound of one on the variables $y_{i t}(37)$ :

$$
\begin{array}{ll}
\operatorname{Min} \sum_{i \in P} \sum_{k=t}^{m-1}\left(s c_{i t}+c v_{i t k}-\pi_{i t}+\pi_{i, k+1}\right) z v_{i t k}+\sum_{i \in P}\left(s c_{i t}+c v_{i t m}-\pi_{i t}\right) z v_{i t m} \\
\sum_{i \in P} \sum_{k=t}^{m}\left(s t_{i t}+v t_{i t} s d_{i t k}\right) z v_{i t k} \leq c a p_{t} & \\
\sum_{k=t}^{m} z v_{i t k} \leq 1 & \forall i \in P \\
z v_{i t k} \geq 0 & \forall i \in P, \forall k \in T \mid k \geq t
\end{array}
$$


The classes in LMCKP correspond to the different items. The inequality constraint (37) can be transformed into a multiple choice equality constraint by including an artificial variable in each class with weight and capacity usage equal to zero. This variable is essentially the slack variable of the original constraint (37).

We develop a branch-and-bound algorithm to solve the subproblem (19)-(22). At each node a problem of the LMCKP type is solved. We use a greedy algorithm [13], which solves the LMCKP optimally. The LMCKP structure remains after branching. Define:

B1 : Set of items for which the set up variable is set to one during branching,

B0 : Set of items for which the set up variable is set to zero during branching,

NB : Set of items for which no branching decision has been made yet,

$$
=P \backslash\{B 1 \cup B 0) \text {. }
$$

After branching, the problem looks as follows:

$$
\begin{array}{lc} 
& \operatorname{Min} \sum_{i \in B 1} s c_{i t}+\sum_{i \in B 1} \sum_{k=t}^{m-1}\left(c v_{i t k}-\pi_{i t}+\pi_{i, k+1}\right) z v_{i t k}+\sum_{i \in B 1}\left(c v_{i t m}-\pi_{i t}\right) z v_{i t m} \\
& +\sum_{i \in N B} \sum_{k=t}^{m-1}\left(s c_{i t}+c v_{i t k}-\pi_{i t}+\pi_{i, k+1}\right) z v_{i t k}+\sum_{i \in N B}\left(s c_{i t}+c v_{i t m}-\pi_{i t}\right) z v_{i t m} \\
\text { s.t. } \quad \sum_{i \in B 1} \sum_{k=t}^{m}\left(v t_{i t} s d_{i t k}\right) z v_{i t k}+\sum_{i \in N B} \sum_{k=t}^{m}\left(s t_{i t}+v t_{i t} s d_{i t k}\right) z v_{i t k} \leq c a p_{t}-\sum_{i \in B 1} s t_{i t} \\
\sum_{k=t}^{m} z v_{i t k} \leq 1 & \forall i \in B 1, \forall i \in N B \\
\sum_{k=t}^{m} z v_{i t k} \leq 0 & \forall i \in B 0 \\
z v_{i t k} \geq 0 & \forall i \in P, \forall k \in T \mid k \geq t
\end{array}
$$

For the set up variables of the items for which no branching decision has been made yet $(i \in N B)$, the value of the set up variable is calculated according to: $\sum_{k=t}^{m} z v_{i t k}=y_{i t}$. In an optimal solution of the LMCKP there is at most one class with variables that have a fractional value, called the fractional class, and at most two variables are fractional in that class [13]. Therefore, there is at most one fractional $y_{i t}$ variable at any node of the $\mathrm{B} \& \mathrm{~B}$ tree. If there is such a fractional $y_{i t}$ variable, we first investigate the branch where we set the fractional $y_{i t}$ variable equal to one. In the twin we set the 
$y_{i t}$ variable, and hence all the production variables in that class, to zero. We backtrack if we find an integer solution. A depth-first strategy is used for selecting the next node. Our problem has some special properties that help to speed up the algorithm:

1. The capacity usage coefficients $v t_{i t} s d_{i t k}$ and $s t_{i t}+v t_{i t} s d_{i t k}$ in (40) are always positive. For the minimization of (39)-(43) at any node of the branch-andbound tree, all variables with a positive objective value coefficient can be set to zero.

2. In the first step of the greedy algorithm, we have to order the variab les in each class by increasing capacity usage. However, the variables $z v_{i t k}$ within a class $i$ for period $t$, are already ordered by weakly increasing capacity usage:

$$
s t_{i t}+v t_{i t} s d_{i t k} \leq s t_{i t}+v t_{i t} s d_{i t, k+1} \quad \forall k \in T \mid t \leq k<m
$$

3. In a further step of the greedy algorithm, we have to determine for each class the set of LP-dominated variables, i.e. variables which will have a value of zero in the optimal LP solution. For the remaining variables in each class, we calculate the slope between adjacent variables in each class using the cost and capacity coefficients. These calculations are done at the root node, but this information is stored and can be re-used further down in the tree. Only when we branch the set up variable of a specific item to one, the cost and capacity coefficients are changed and we have to do these calculations again for this class.

\section{Computational Results}

\subsection{Lower Bound Calculation with Lagrange Relaxation}

We implement a Lagrange relaxation scheme where the dual prices are pdated by subgradient optimization, as described in the previous section. We test our procedure on the $\mathrm{F}$ and $\mathrm{G}$ test sets from Trigeiro et al. [14]. The $\mathrm{F}$ test set contains 70 problems with 6 products and 15 periods. The G set contains 71 problems with 6,12 and 24 products and 15 and 30 periods. There are 5 problems for each combination, except for the combination of 6 products and 15 periods for which there are 46 problems. 
Our algorithm is coded in Fortran and the tests are done on a Pentium $750 \mathrm{MHz}$ computer. We initialize the dual prices at zero $\left(p_{i t}^{0}=0\right)$. Further we set $\omega$ in equation (34) equal to two, and decrease it by multiplying it with the parameter $r f$, which was set at 0.9 , if the Lagrange solution $\bar{v}_{E M L R}\left(p_{i t}^{r}\right)$ did not improve in the last 50 iterations. We use the Trigeiro et al. [14] (TTM) heuristic to quickly obtain an upper bound (UB) which we use in the updating formula (34). The subproblem is solved with our specialized branch-and-bound algorithm. Table 2 reports the gap which is calculated as $100 * \frac{\text { upperbound-lower bound }}{\text { lower bound }}$. The upper bounds we use are obtained separately from an algorithm using column generation with the first decomposition, i.e. per item, as described in Jans [8]. As we use the same upper bounds, the differences in the gaps are only due to the differences in the lower bounds. In the column TTM, we report the gap compared to the lower bound obtained by the Trigeiro et al. algorithm [14], which uses Lagrange relaxation dualizing the capacity constraints in formulation (1)-(5). The column DW1 refers to the gap of the upper bound relative to $\bar{v}_{C L D 1}$, the lower bound obtained with the first decomposition on the original formulation, as reported in [8]. The other columns give the gap with the best Lagrange lower bound for the new decomposition after 500, 1000, 2000, 3000, 4000 and 5000 iterations. For the problems with 15 time periods, we need 2000 iterations before we obtain a gap which is better than the gap with the lower bound of the first decomposition. For the problems with 30 products we need 3000 iterations, and even 4000 for the most difficult set, before we obtain better gaps. In the column ' $\%$ change' we report the relative decrease in gap after 5000 Lagrange iterations, compared to DW1. It seems that the relative improvement in the gap decreases as we increase the number of products and periods. For problems with many products, the first decomposition already gives a good lower bound [9], so there might be less opportunity for improvement. 
Table 2. Comparison of gaps (\%)

\begin{tabular}{l|rrrrrrrrr} 
& TTM & $\mathbf{5 0 0}$ & $\mathbf{1 0 0 0}$ & $\mathbf{2 0 0 0}$ & $\mathbf{3 0 0 0}$ & $\mathbf{4 0 0 0}$ & $\mathbf{5 0 0 0}$ & $\mathbf{D W 1}$ & $\begin{array}{c}\% \\
\text { change }\end{array}$ \\
\cline { 2 - 9 } $\mathbf{F 6 - 1 5}$ & & & & & & & & & \\
G6-15 & 4.60 & 6.80 & 4.34 & 3.26 & 3.05 & 2.99 & 2.98 & 3.55 & 16.13 \\
$\mathbf{G 1 2 - 1 5}$ & 1.11 & 2.13 & 1.29 & 1.02 & 0.96 & 0.95 & 0.95 & 1.07 & 11.92 \\
$\mathbf{G 2 4 - 1 5}$ & 0.36 & 0.67 & 0.45 & 0.35 & 0.33 & 0.32 & 0.32 & 0.36 & 11.51 \\
$\mathbf{G 6 - 3 0}$ & 3.22 & 12.09 & 5.10 & 3.33 & 2.97 & 2.89 & 2.86 & 3.22 & 11.03 \\
$\mathbf{G 1 2 - 3 0}$ & 1.15 & 5.28 & 2.34 & 1.26 & 1.10 & 1.05 & 1.04 & 1.15 & 9.57 \\
$\mathbf{G 2 4 - 3 0}$ & 0.24 & 4.14 & 1.40 & 0.37 & 0.26 & 0.23 & 0.22 & 0.24 & 7.57
\end{tabular}

In Table 3, we give the CPU times for TTM, for performing the new Lagrange relaxation with 500, 1000, 2000, 3000, 4000 and 5000 iterations and for DW1. Remark that the CPU times for the new Lagrange relaxation and DW1 include the time for performing the TTM heuristic. We observe that the CPU time increases with increasing number of periods and products. The CPU times for the Lagrange decomposition per period are considerably higher compared the Lagrange and Dantzig-Wolfe decomposition per item. Further we implemented the Lagrange relaxation using the LINDO solver [12] to optimize the subproblems and the CPU times for the $\mathrm{F}$ test set with this implementation are given in the row F-lindo, indicating the superiority of our subproblem algorithm.

Table 3. Comparison of CPU times in seconds

\begin{tabular}{|c|c|c|c|c|c|c|c|c|}
\hline & TTM & 500 & 1000 & 2000 & 3000 & 4000 & 5000 & DW1 \\
\hline F6-15 & 0.16 & 0.78 & 1.65 & 3.82 & 6.36 & 9.11 & 11.92 & 0.28 \\
\hline G6-15 & 0.17 & 0.70 & 1.41 & 3.24 & 5.45 & 7.85 & 10.34 & 0.33 \\
\hline G12-15 & 0.29 & 1.78 & 4.16 & 10.67 & 19.29 & 29.01 & 39.09 & 0.56 \\
\hline G24-15 & 0.55 & 6.41 & 17.01 & 45.13 & 82.24 & 125.07 & 171.00 & 1.10 \\
\hline G6-30 & 0.65 & 1.86 & 3.65 & 8.40 & 14.27 & 20.78 & 27.53 & 1.06 \\
\hline G12-30 & 1.28 & 3.95 & 8.67 & 23.12 & 43.74 & 69.00 & 97.19 & 2.12 \\
\hline G24-30 & 2.52 & 7.39 & 15.73 & 45.10 & 93.34 & 159.76 & 245.07 & 4.66 \\
\hline F-lindo & - & 21.04 & 50.60 & 133.21 & 246.86 & 379.55 & 519.95 & - \\
\hline
\end{tabular}

We also experimented with some other settings for the initial dual prices and the $r f$ parameter. Initializing the dual prices at the set up cost $\left(p_{i t}^{0}=s c_{i}\right)$ gives results that are very similar to the case where the initial dual prices are zero. Initializing the dual 
prices with the following formula, $p_{i t}^{0}=s c_{i} *(m-t+1)$ increases the CPU time while the quality of the gap decreases. Except for the simplest problems with 6 products and 15 periods, we cannot improve the lower bound of the first decomposition. Initializing the dual prices at zero $\left(p_{i t}^{0}=0\right)$ but decreasing the multiplier $r f$ to 0.8 slightly increases both the CPU time and the gap. Decreasing $r f$ further to 0.7 increases the final gaps even further. Also increasing $r f$ to 0.95 or 0.99 results in worse gaps after 5000 iterations. Faster initial convergence in the first 500 iterations can be obtained

by changing the $r f$ parameters to 0.5 and changing the stepsize if no improvement is obtained in the last 20 steps. However, in later iterations the solution does not improve anymore as the stepsize becomes too small.

\subsection{Comparison with Other Lower Bounds in the Literature}

Finally, in Table 4 we give specific lower bound results for the 6 data sets that are used in Belvaux and Wolsey [2] and Miller et al. [10]. For the data set with 6 products and 15 periods, G30 is the original data set with fractional variable production times as used by Trigeiro et al. [14], whereas G30b is this data set with unit variable production times as used by Belvaux and Wolsey. All the other data sets have unit variable production times. First we report the Lagrange lower bound, as obtained after 300 iterations of the Trigeiro et al. algorithm (TTM). Next, DW1 is the DantzigWolfe lower bound of the first decomposition on the regular formulation, where the problem decomposes into subproblems per item. These results are taken from Jans [8]. Belvaux and Wolsey (BW) present a branch-and-cut algorithm, consisting of both general cutting planes and specific lot sizing inequalities. Compared to DW1, they obtain better bounds for the smaller problems and the same bounds for the larger problems. Miller et al. [10] (MNS) derive cuts for the 'Single Period relaxation with Preceding Inventory'. This is a one period model, taking into account the demand for all items in that period and the capacity constraint. They improve the lower bounds obtained by Belvaux and Wolsey, and their bounds are, to the best of our knowledge, the best lower bounds for this problem reported in the literature so far. Finally, we give the results for the second decomposition on the network reformulation, where the problem is decomposed into subproblems per period. We present the lower bounds 
obtained by Lagrange Relaxation (LAG2). The Lagrange lower bounds domin ate the MNS lower bounds on these test problems. We also calculated the optimal lower bound with Dantzig -Wolfe decomposition and column generation (DW2). These last results (DW2) are taken from Jans [8]. However, this algorithm is only able to solve the smallest problems successfully.

Table 4. Comparison of lower bounds

\begin{tabular}{l|cccccc} 
& TTM & DW1 & BW & MNS & LAG2 & DW2 \\
\hline $\operatorname{Tr} 6-15(G 30)$ & $37,102.8$ & $37,103.1$ & - & - & $37,426.9$ & $37,431.4$ \\
$\operatorname{Tr} 6-15(G 30 b)$ & $37,198.6$ & $37,201.2$ & $37,213.3$ & 37,319 & $37,380.3$ & $37,382.0$ \\
$\operatorname{Tr} 6-30(G 62)$ & $60,946.1$ & $60,946.2$ & $60,979.4$ & 61,150 & $61,189.1$ & $61,204.8$ \\
$\operatorname{Tr} 12-15(G 53)$ & $73,756.8$ & $73,847.9$ & $73,858.2$ & 73,929 & $73,942.7$ & $73,944.5$ \\
$\operatorname{Tr} 12-30(G 69)$ & $130,176.9$ & $130,177.2$ & 130,177 & 130,292 & $130,330.4$ & $130,337.7$ \\
$\operatorname{Tr} 2415(G 57)$ & $136,363.7$ & $136,365.7$ & 136,366 & 136,388 & $136,417.9$ & - \\
$\operatorname{Tr} 2430(G 72)$ & $287,753.2$ & $287,753.4$ & 287,753 & 287,811 & $287,824.3$ & -
\end{tabular}

In Table 5, we also give the CPU times for calculating the lower bounds with the different methods. Belvaux and Wolsey and Miller et al. do not report the individual CPU times for calculating their lower bounds at the root node, but Miller et al. state that the time for calculating this lower bound never exceeds a few seconds on a 350 $\mathrm{MHz}$ computer for these test problems. We observe that the CPU times for the second decomposition are much larger compared to the first decomposition. This is true for both the Lagrange implementation and the Dantzig-Wolfe decomposition.

Table 5. CPU times for lower bounds

\begin{tabular}{l|cccccc} 
& TTM & DW1 & BW & MNS & LAG2 & DW2 \\
\hline Tr6-15 (G30) & 0.22 & 0.33 & - & - & 8.49 & 1.44 \\
$\operatorname{Tr} 6-15(G 30 b)$ & 0.20 & 0.38 & - & - & 14.93 & 1.64 \\
$\operatorname{Tr} 6-30(G 62)$ & 0.66 & 0.88 & - & - & 16.71 & 9.1 \\
$\operatorname{Tr} 12-15(G 53)$ & 0.38 & 0.82 & - & - & 30.63 & 25.58 \\
$\operatorname{Tr} 12-30(G 69)$ & 1.49 & 1.82 & - & - & 74.50 & 374.57 \\
$\operatorname{Tr} 2415(G 57)$ & 0.72 & 0.99 & - & - & 194.04 & - \\
$\operatorname{Tr} 2430(G 72)$ & 3.41 & 3.85 & - & - & 290.49 & -
\end{tabular}




\section{Conclusions}

We present a Dantzig-Wolfe reformulation that gives an equal or better lower bound compared to the textbook decomposition approach for the Capacitated Lot Sizing Problem with Set Up Times. The network reformulation of the lot sizing problem is decomposed into subproblems per time period, whereas the textbook decomposition on the original formulation leads to uncapacitated subproblems per item. In the new decomposition, the subproblems contain the setup constraints and a capacity constraint. We develop a specialized branch-and-bound algorithm for the subproblem using properties of the Linear Multiple Choice Knapsack Problem. Computational experiments confirm that we obtain improved lower bounds. A comparison with the literature indicates that these bounds are better than both the bounds obtained by Belvaux and Wolsey [2] and Miller et al. [10] for the data sets reported in these papers. The experiments also show that the CPU time for obtaining these bounds is considerable. 


\section{References}

[1] BARANY, I., T.J. VAN ROY AND L.A. WOLSEY, Uncapacitated Lot-Sizing: the Convex Hull of Solutions, Mathematical Programming Study 22 (1984) $32-43$.

[2] BELVAUX, G., AND L.A. WOLSEY, BC-PROD: A Specialized Branch-and-Cut System for Lot-Sizing Problems, Management Science 46 (5) (2000) 724-738.

[3] CHEN, W.H. AND J.M. THIZY, Analysis of Relaxations for the Multi-Item Capacitated Lot-Sizing Problem, Annals of Operations Research 26 (1990) 29-72.

[4] DIABY, M., H.C. BAHL, M.H. KARWAN, AND S. ZIONTS, Capacitated LotSizing and Scheduling by Lagrangean Relaxation, European Journal of Operational Research 59 (1992) 444-458.

[5] DZIELINSKI B.P. AND R.E. GOMORY, Optimal Programming of Lot Sizes, Inventory and Labor Allocations, Management Science 11 (9) (1965) 874-890.

[6] EPPEN, G.D., AND R.K. MARTIN, Solving Multi-Item Capacitated Lot-Sizing Problems Using Variable Redefinition, Operations Research 35 (6) (1987) 832-848.

[7] FISHER, M.L., An Applications Oriented Guide to Lagrangian Relaxation, Interfaces 15 (2) (1985) 10-21.

[8] JANS, R., Capacitated Lot Sizing Problems: New Applications, Formulations and Algorithms, PhD. Thesis (2002), K.U.Leuven, Belgium, 205 p.

[9] MANNE, A.S., Programming of Economic Lot Sizes, Management Science 4 (2) (1958) 115- 135.

[10] MILLER, A.J., G.L. NEMHAUSER AND M.W.P. SAVELSBERGH, Solving Multi-Item Capacitated Lot-Sizing Problems with Setup Times by Branchand-Cut, CORE Discussion paper 2000/39 (2000) 32 p.

[11] PISINGER, D., A Minimal Algorithm for the Multiple-Choice Knapsack Problem, European Journal of Operational Research 83 (1995) 394-410.

[12] SCHRAGE, L., LINDO: Optimization software for Linear Programming, Lindo Systems Inc., Chicago, IL. (1995)

[13] SINHA, P. AND A.A. ZOLTNERS, The Multiple-Choice Knapsack Problem, Operations Research 27 (3) (1979) 503-515. 
[14] TRIGEIRO, W., L.J. THOMAS, AND J.O. McCLAIN, Capacitated Lot Sizing with Set-Up Times, Management Science 35 (3) (1989) 353-366.

[15] WAGNER H.M. AND T.M. WHITIN, Dynamic Version of the Economic Lot Size Model, Management Science 5 (1) (1958) 89-96.

Appendix: Proof of proposition 2: $\bar{v}_{C L D 2} \leq \bar{v}_{E M D 2}$

We first prove that a feasible solution of the decomposition of the network model per period corresponds to a feasible solution of the decomposition per period of the regular formulation with the same objective value. Next we prove that the reverse is not necessarily true. The proof is similar to the one given in [3] for the CLSP without set up times.

Let $\left(z v_{i t k}, w_{i t}, y_{i t}\right)$ be a feasible solution of the decomposition model per period of the network formulation. This implies that the solution 1) satisfies the network constraints (7) and (8) and 2) it is contained in the convex hull defined by the constraints (9)-(12).

Let $E_{t}$ be the set of all the extreme points $\left(z v_{i t k}^{e}, y_{i t}^{e}\right)$ of the convex hull of (9)-(12) for period $t$. The feasible solution $\left(z v_{i t k}, w_{i t}, y_{i t}\right)$ can then be expressed as a convex combination of these extreme points:

$$
\begin{aligned}
z v_{i t k}=\sum_{e=1}^{\left|E_{t}\right|} \lambda_{t e} z v_{i t k}^{e} \text { and } y_{i t}=\sum_{e=1}^{\left|E_{t}\right|} \lambda_{t e} y_{i t}^{e}, \text { with } \sum_{e=1}^{\left|E_{t}\right|} \lambda_{t e}=1, \lambda_{t e} \geq 0 & \\
& \forall i \in P, \forall t \in T, \forall k \in T, k \geq t
\end{aligned}
$$

For the feasible point $\left(z v_{i t k}, w_{i t}, y_{i t}\right)$ we can find the according solution in the $\left(x_{i t}, s i_{i}, s_{i t}, y_{i t}\right)$-space as follows [6]:

$$
\begin{array}{ll}
x_{i t}=\sum_{k=t}^{m} z v_{i t k} s d_{i t k}=\sum_{k=t}^{m}\left(\sum_{e=1}^{\left|E_{t}\right|} \lambda_{t e} z v_{i t k}^{e}\right) s d_{i t k} & \forall i \in P, \forall t \in T \\
y_{i t}=y_{i t} & \forall i \in P, \forall t \in T \\
s_{i}=\sum_{k=1}^{m} w_{i k} s d_{i 1 k} & \forall i \in P \\
s_{i t}=s_{i}+\sum_{k=1}^{t}\left(x_{i k}-d_{i k}\right) & \forall i \in P, \forall t \in T
\end{array}
$$


For each of the extreme points $\left(z v_{i t k}^{e}, y_{i t}^{e}\right)$ of the convex hull of (9)-(12), we can also find the according solution in the $\left(x_{i t}, y_{i t}\right)$-space in the same way:

$$
x_{i t}^{e}=\sum_{k=t}^{m} z v_{i t k}^{e} s d_{i t k}, y_{i t}^{e}=y_{i t}^{e}
$$

The solution $\left(x_{i t}^{e}, y_{i t}^{e}\right)$ satisfies constraint (3)-(5), as the original solution $\left(z v_{i t k}^{e}, y_{i t}^{e}\right)$ satisfies (9)-(12).

Now we have that $x_{i t}=\sum_{k=t}^{m} \sum_{e=1}^{\left|E_{t}\right|} \lambda_{t e} z v_{i t k}^{e} s d_{i t k}=\sum_{e=1}^{\left|E_{t}\right|} \lambda_{t e} x_{i t}^{e}$ and $y_{i t}=\sum_{e=1}^{\left|E_{t}\right|} \lambda_{t e} y_{i t}^{e}$. The solution $\left(x_{i t}, s i_{i}, s_{i t}, y_{i t}\right)$ is in the convex hull of constraints (3)-(5), as it is a convex combination of feasible solutions $\left(x_{i t}^{e}, y_{i t}^{e}\right)$ satisfying (3)-(5). As the original solution $\left(z v_{i t k}, w_{i t}, y_{i t}\right)$ satisfies the flow equations, the according solution $\left(x_{i t}, s i_{i}, s_{i t}, y_{i t}\right)$ satisfies the demand equations (2.1) and (2.2). Therefore $\left(x_{i t}, s i_{i}, s_{i t}, y_{i t}\right)$ is a feasible solution for the decomposition per period of the regular formulation. The definition of the cost coefficients in the network reformulation ensures that both solutions have the same objective value.

Chen and Thizy [3] give an example where $\bar{v}_{C L D 2}<\bar{v}_{C L D 1}$ and from Proposition 1 we know that $\bar{v}_{C L D 1} \leq \bar{v}_{E M D 2}$. Hence a feasible point $\left(x_{i t}, s i_{i}, s_{i t}, y_{i t}\right)$ for the decomposition model per period of the regular formulation is not necessarily feasible for the decomposition model per period of the network formulation. Q.E.D. 


\section{Publications in the Report Series Research* in Management}

ERIM Research Program: "Business Processes, Logistics and Information Systems"

\section{3}

Project Selection Directed By Intellectual Capital Scorecards

Hennie Daniels and Bram de Jonge

ERS-2003-001-LIS

http://hdl.handle.net/1765/265

Combining expert knowledge and databases for risk management

Hennie Daniels and Han van Dissel

ERS-2003-002-LIS

http://hdl.handle.net/1765/266

Recursive Approximation of the High Dimensional max Function

Ş. II. Birbil, S.-C. Fang, J.B.G. Frenk and S. Zhang

ERS-2003-003-LIS

http://hdl.handle.net/1765/267

Auctioning Bulk Mobile Messages

S.Meij, L-F.Pau, E.van Heck

ERS-2003-006-LIS

http://hdl.handle.net/1765/274

Induction of Ordinal Decision Trees: An MCDA Approach

Jan C. Bioch, Viara Popova

ERS-2003-008-LIS

http://hdl.handle.net/1765/271

A New Dantzig-Wolfe Reformulation And Branch-And-Price Algorithm For The Capacitated Lot Sizing Problem With Set Up Times

Zeger Degraeve, Raf Jans

ERS-2003-010-LIS

http://hdl.handle.net/1765/275

Reverse Logistics - a review of case studies

Marisa P. de Brito, Rommert Dekker, Simme D.P. Flapper

ERS-2003-012-LIS

http://hdl.handle.net/1765/277

Product Return Handling: decision-making and quantitative support

Marisa P. de Brito, M. (René) B. M. de Koster

ERS-2003-013-LIS

http://hdl.handle.net/1765/278

* A complete overview of the ERIM Report Series Research in Management: $\underline{\text { http://www.erim.eur.nl }}$

ERIM Research Programs:

LIS Business Processes, Logistics and Information Systems

ORG Organizing for Performance

MKT Marketing

F\&A Finance and Accounting

STR Strategy and Entrepreneurship 
Managing Product Returns: The Role of Forecasting

Beril Toktay, Erwin A. van der Laan, Marisa P. de Brito

ERS-2003-023-LIS

http://hdl.handle.net/1765/316

Improved Lower Bounds For The Capacitated Lot Sizing Problem With Set Up Times

Zeger Degraeve, Raf Jans

ERS-2003-026-LIS

In Chains? Automotive Suppliers and Their Product Development Activities

Fredrik von Corswant, Finn Wynstra, Martin Wetzels

ERS-2003-027-LIS

Mathematical models for planning support

Leo G. Kroon, Rob A. Zuidwijk

ERS-2003-032-LIS

How and why communications industry suppliers get "squeezed out" now, and the next phase

L-F Pau

ERS-2003-033-LIS

http://hdl.handle.net/1765/317

Financial Markets Analysis by Probabilistic Fuzzy Modelling Jan van den Berg, Uzay Kaymak, Willem-Max van den Bergh ERS-2003-036-LIS

http://hdl.handle.net/1765/323

WLAN Hot Spot services for the automotive and oil industries :a business analysis or : "Refuel the car with petrol and information , both ways at the gas station "

L-F Pau, M.H.P.Oremus

ERS-2003-039-LIS

A Lotting Method for Electronic Reverse Auctions

U. Kaymak, J.P. Verkade and H.A.B. te Braake

ERS-2003-042-LIS 